\title{
PLC based model predictive control for industrial process control
}

Sohaib Aslam*, Sundas Hannan, Muhammad Umar Sajjad, Muhammad Waheed Zafar

Department of Electrical Engineering, Faculty of Engineering and Technology, Superior University, Lahore, Pakistan

\section{ART ICLE INFO}

\section{Article history:}

Received 3 January 2017

Received in revised form

24 March 2017

Accepted 31 March 2017

\section{Keywords:}

Programmable logic controller

Model predictive control

Pulse width modulation

Liquid tank

State-space model

\begin{abstract}
A B S T R A C T
This paper presents the real-time implementation of model predictive control technique on programmable logic controller for industrial process control. Firstly, temperature of water is regulated in liquid tank at desired temperature and then level of water in filling bottles is controlled through PWM based flow control valve. The simulations of MPC for temperature and level control are presented in MATLAB by first developing the system model of water tank. The simulation results have shown that MPC effectively regulates both temperature and level of water. Finally, MPC is real time implemented on PLC and provides better real-time control of these two variables.
\end{abstract}

(C) 2017 The Authors. Published by IASE. This is an open access article under the CC BY-NC-ND license (http://creativecommons.org/licenses/by-nc-nd/4.0/).

\section{Introduction}

In the industrial automation field, various hardware and software architectures and accesses are currently in use for implementing control applications (Colla et al., 2009). To fulfill high control performance requirements in industry the control methods are mostly based on the proportional, integral and derivative (PID) controller which is widely used since the last few decades (Rathore et al., 2015). It is combination of three terms Proportional, Integral and Derivative and it is mostly used to influence the time-domain response of vast range of dynamic systems (Aslam et al., 2016a). Because of its reliability and tuning issues using it in different environments is a challengeable task and most of the control processes operate at constraints. PID is not abile to work within the predetermined constraints without violating them (Aslam et al., 2016a; Huyck et al., 2012). Model Predictive Control (MPC) is the solution for that and has become a widely-applied control technique in process industry for the control of slow dynamic systems i.e. chemical industries, paper industries etc. The key feature of MPC is to repeatedly solve an optimization problem based on available measurements of the current state of the process (Huyck et al., 2012).One critical challenge in MPC implementation is the high computational burden required for optimization of control signal and even it becomes more critical for

\footnotetext{
* Corresponding Author.

Email Address: sohaib_engg2000@yahoo.com (S. Aslam) https://doi.org/10.21833/ijaas.2017.06.009

2313-626X/C) 2017 The Authors. Published by IASE.

This is an open access article under the CC BY-NC-ND license

(http://creativecommons.org/licenses/by-nc-nd/4.0/)
}

systems with fast sampling time (Li et al., 2013). Mostly embedded systems have limited computational power and it is not feasible to implement MPC on it (Maciejowski, 2002). Programmable Logic Controller (PLC) has high computational power and it provides best choice to implement MPC. PLC can be programmed in numerous programming languages i.e. Function Block Diagram (FBD), Statement List (STL), and Ladder Logic Diagram (LAD). LAD is the most famous PLC programming language and it uses components like elements used in a line diagram format to describe hard-wired control (Lashin, 2014). In this research work simulations of MPC are presented for temperature and level control at different set-points by first developing the state space model of liquid tank for both temperature and level control systems and finally MPC is real-time implemented on PLC by using ladder logic programming for both temperature and level control in tank and bottles respectively and the results are displayed on HMI. In section 2 system modeling and implementation of MPC is done. Section 3 elaborates the PLC operation. Section 4 and 5 presents the simulation results and hardware implementation respectively. Finally conclusion is presented in section 6.

\section{Implementation of MPC}

Model predictive control (MPC) refers to a class of control algorithms that utilizes process model to predict the future response of a plant. At each sampling interval MPC attempts to optimize future plant behavior by computing a sequence of future controlled inputs. The first input in the optimal 
sequence is applied to the plant while rest of sequence is ignored and this entire calculation is repeated at every sampling instant (Qin and Badgwell, 2003). The basic structure of MPC is shown in Fig. 1.

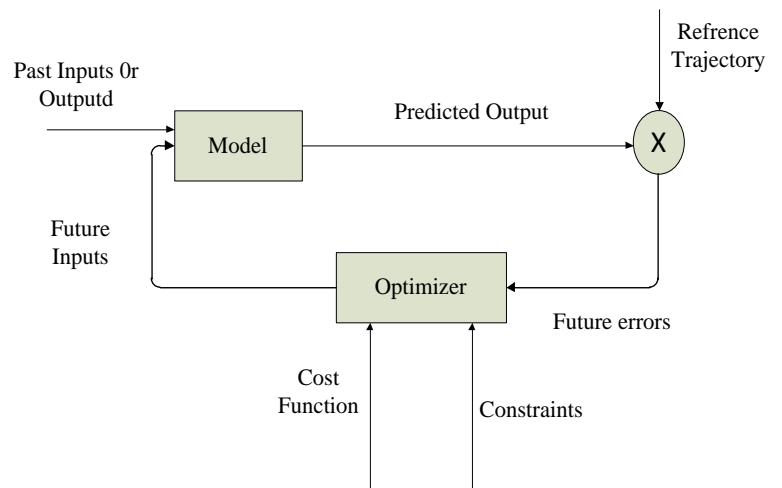

Fig. 1: Basic structure of MPC

Implementation of MPC is based on system model and optimizer. Optimizer is a mathematical function which optimizes the control signal by minimizing the cost function in presence of constraints. These control signals as future inputs are send to system model of the plant to get predicted output. The difference between the predicted output and reference value in form of errors is sent to optimizer which optimizes the future input and this procedure will remain continue.

\subsection{System modeling}

Perfection of MPC depends on how well dynamics of a system have been captured. In this research work two industrial process variables temperature and level are controlled. The system model of whole process is shown in Fig. 2. MPC is implemented on PLC to regulate these two variables. Resistive heating element is used as an actuator to provide heat to liquid tank and PT100 thermocouple is used to measure temperature and provides feedback to PLC. After acquiring the desired temperature of water filling of bottles start and flow of water is controlled by regulating the Pulse Width Modulation (PWM) based flow control valve, while level of water is measured by weight cell as $1 \mathrm{Kg}$ of water is equal to 1 liter of water therefore weight cell is used to measure level and provides feedback to PLC. The State Space Model (SSM) of liquid tank for temperature control and level control are presented below.

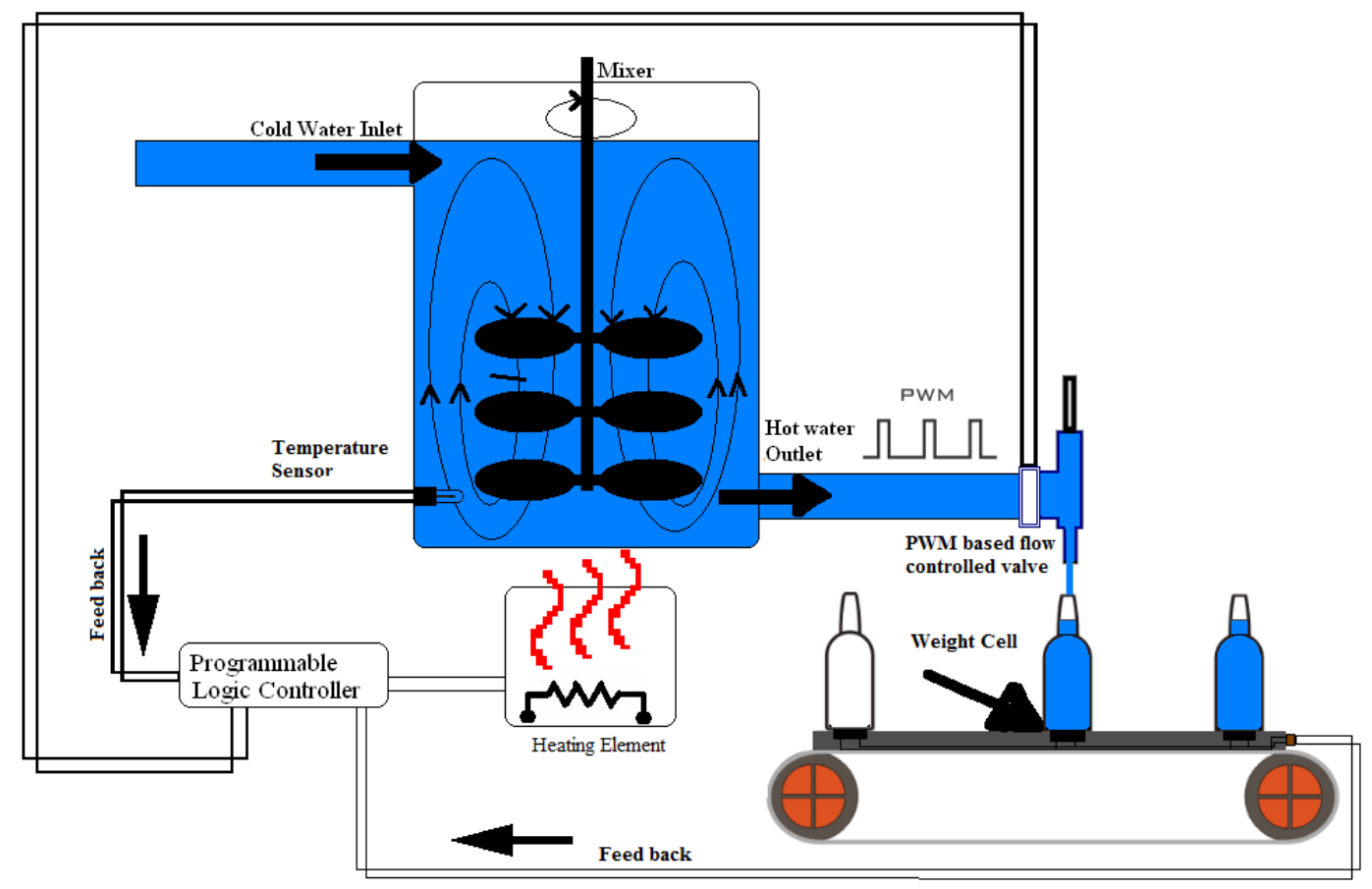

Fig. 2: System's model

\subsubsection{System model for temperature control}

The performance of heating process depends on the induction, maintenance and control. There are number of heating techniques used now a day in domestic and industrial processes i.e. direct heating, indirect heating, electric arc heating etc. (Aslam et al., 2016a). In this research work direct resistance heating technique is employed in which immersion rod is dipped in the water tank. The heating capacity of immersion rod is based on its wattage power and the required wattage power for the immersion rod is calculated by the equation (Liuping, 2009) (Eq. 1):

$Q_{T}=\frac{W C \Delta T_{\text {water }}}{3412 . T}$

where $Q_{T}$ is the heater wattage, $W$ is the weight of water, $C$ is the specific heat of water, $\Delta T$ is the change in temperature, and $T$ is the time required to raise temperature at desired level. In this case $W$ is 
33lb, $C_{P}$ is $1 \mathrm{Btu} / \mathrm{lb} / \mathrm{F}, \Delta T$ is $140 \mathrm{~F}$, and $T$ is 1 hours. By putting the values in above equation, the required wattage is calculated as:

$Q_{T}=\frac{88 * 1 * 140}{3412 * 1}=1.8 \mathrm{KW}$

The required power of immersion rod is $1.8 \mathrm{KW}$ so $2 \mathrm{KW}$ heating rod is selected. This immersion rod is used to regulate the temperature of water tank system by using MPC. Effectiveness of MPC is based on the precision of system model of liquid tank Performance of MPC depends on how well the dynamics of system have been captured. The system model of water tank is shown in Fig. 3 (Aslam et al., 2016b).

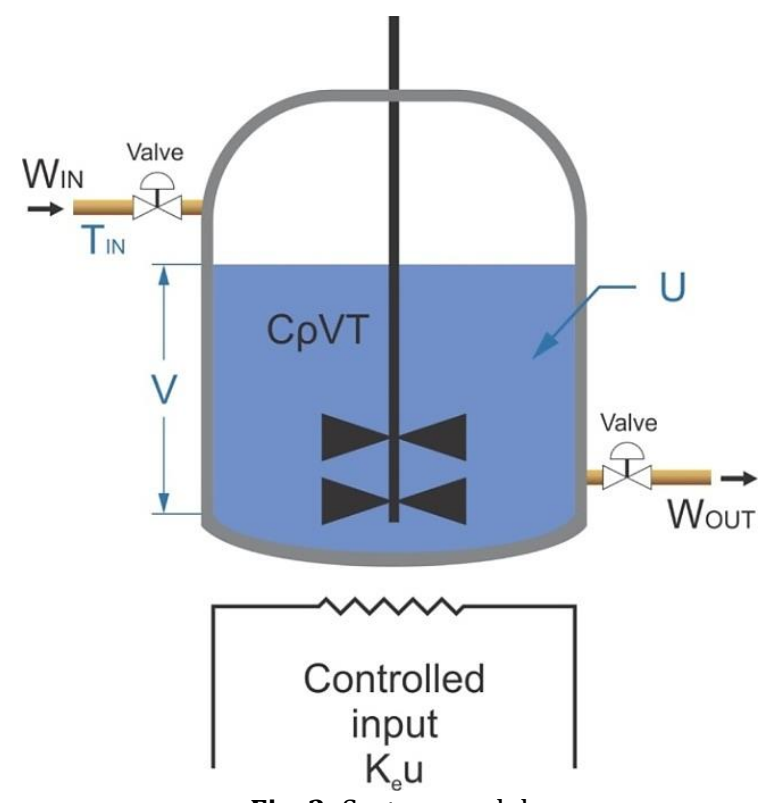

Fig. 3: System model

Where $K_{e} u$ is the input power, $\mathrm{C} \rho V T_{1}$ is the energy in the tank, $\rho$ is the density of water, $V$ is the volume, $T_{1}$ is the water temperature in the tank, $T_{\text {in }}$ is the inlet temperature, $U$ is the thermal conductivity, and $W$ is the mass flow rate of water. The parameter values of liquid tank are shown in Table 1.

Table 1: Parameters of liquid tank for temperature control

\begin{tabular}{ccc}
\hline Number & Parameter & Value \\
\hline 1 & Weight & $\mathrm{K}_{\text {tau }}=40 \mathrm{Kg}$ \\
2 & Inlet Flow rate & $\mathrm{W}_{\text {in }}=20 \mathrm{Kg} / \mathrm{min}$ \\
3 & Input Power & $\mathrm{K}_{\mathrm{e}}=2 \mathrm{KW}$ \\
4 & Specific Heat Capacity & $\mathrm{C}=4200 \mathrm{~J} / \mathrm{Kg}-\mathrm{K}$ \\
5 & Thermal Conductivity & $\mathrm{U}=0.6 \mathrm{~W} / \mathrm{mK} @ 20^{\circ} \mathrm{C}$ \\
6 & Density & $\rho=1000 \mathrm{Kg} / \mathrm{m}^{3}$ \\
7 & Volume & $\mathrm{V}=0.20 \mathrm{~m}^{3}$ \\
\hline
\end{tabular}

The thermodynamic equation for water tank is shown below (Eq. 2)

$\frac{d\left(C \rho V T_{1}\right)}{d t}=K_{e} u+c W\left(T_{i n}-T_{1}\right)+U\left(T_{e n v}-T_{1}\right)$

by taking Laplace of above equation it becomes (Eq. 3)

$C \rho V\left[s T_{1}(s)-T_{1 o}\right]=K_{e} u(s)+C W\left(T_{i n}(s)-T_{1}(s)\right)+$ $U\left(T_{\text {env }}(s)-T_{1}(s)\right)$ accepted that $T_{\text {in }}=T_{\text {env }}$ (Eq. 4)

$C \rho V\left[s T_{1}(s)-T_{1 o}\right]=K_{e} u(s)+(C w+U)\left(T_{i n}(s)-T_{1}(s)\right)$

solving for $\mathrm{T}_{1}(\mathrm{~s})$ above equation becomes (Eq. 5)

$T_{1}(s)=\frac{K_{e} / c W+U}{\frac{\rho V}{(w+U / c)} s+1} u(s)+\frac{1}{\frac{\rho V}{(w+U / c)} s+1} T_{i n}(s)+\frac{\rho V /(w+U / c)}{\frac{\rho V}{(w+U / c)} s+1} T_{1 o}$

$\tau$ is the time delay between an excitation of heating element and the response of temperature sensor (Eq. $6)$.

$T(t)=T_{1}(t-\tau)$

apply Laplace transformation to above equation it becomes (Eq. 7)

$T(s)=T_{1}(s) e^{-\frac{K_{\tau}}{W} s}$

by putting $T_{1}(s)$ in above equation, the transfer function becomes (Eq. 8)

$\frac{T(s)}{u(s)}=\frac{K_{e} / c w+U}{\frac{\rho V}{(w+U / c)} s+1} e^{-\frac{K_{\tau}}{w} s}$

here $\tau=\frac{K_{\tau}}{w}, \quad K_{u}=\frac{K_{e}}{c w+U}, \quad T_{k}=\frac{\rho V}{(w+U / c)}$ so transfer function $\mathrm{H}(\mathrm{s})$ becomes (Eq. 9)

$H(s)=\frac{K_{u}}{T_{k} s+1} e^{-\tau s}$

by putting the values of parameters from Table 1 to above equation, transfer function becomes (Eq. 10)

$H(s)=\frac{0.02}{10 s+1} e^{0.03}$

The transfer function obtained is discretized by sampling interval of $10 \mathrm{sec}$ to convert it in discretetime state-space model as shown in equation below (Aslam et al., 2016b) (Eqs. 11 and 12)

$x_{m}(k)=\left[\begin{array}{lll}1 & 0 & 0 \\ 1 & 0 & 0 \\ 0 & 0 & 0\end{array}\right] x_{m}(k)+\left[\begin{array}{c}0.0200 \\ 0 \\ 0\end{array}\right] u(k)$
$y(k)=\left[\begin{array}{lll}1 & 0 & 0\end{array}\right] x_{m}(k)$

The above two equations represent the State Space Model (SSM) of the liquid tank that will be used in Fig. 1 to implement MPC for temperature control of water tank.

\subsubsection{System model for level control}

Fluid level control in a tank is a very common application in process industries. Consider a liquid tank as shown in Fig. 2 with water flowing in and flowing out as well. The output of tank is the level of water represented in terms of height $h$ and input to the system is flow in rate (fin) of water. The flow out 
( $f_{\text {out }}$ ) of water depends on the pressure in the tank as shown by Eq. 13

$f_{\text {out }}=R \rho g h$

Where $\rho$ is density of water, $\mathrm{g}$ is the gravitational acceleration, $\mathrm{h}$ is height of water in tank and $\mathrm{R}$ is the proportionality constant related to outlet pipe shape and for simplicity it is considered 1 and above relation becomes

$f_{\text {out }}=\rho g h$

The rate of change of volume in tank with uniform cross sectional area can be related with rate of change in height of water in tank and also with the difference between flow in and flow out by the Eq. 14.

$\frac{d V}{d t}=A \frac{d h}{d t}=f_{\text {in }}-f_{\text {out }}$

Where, A is uniform cross sectional area of tank. By putting the value of $\mathrm{f}_{\text {out }}$ in Eq. 14 it becomes the first order differential equation represented by Eq. 15

$A \frac{d h}{d t}+\rho g h=f_{\text {in }}$

By dividing the above equation by $\rho g$ the Eq. 15 becomes

$\frac{A}{\rho g} \frac{d h}{d t}+h=\frac{f_{\text {in }}}{\rho g}$

The above relation can be represented in terms of time constant $\tau$ and gain $\mathrm{K}$ by the following equation

$\tau \frac{d h}{d t}+h=K f_{\text {in }}$

Where, $\tau=\frac{A}{\rho g}$ and $K=\frac{1}{\rho g}$. The transfer function $1^{\text {st }}$ order model of the system is shown in Eq. 18

$H(s)=\frac{\frac{1}{\rho g}}{\frac{A}{\rho g} S+1}$

The parameters of liquid tank for system model of level control are shown in Table 2. By putting the values of parameters in Eq. 18 the actual transfer function for level control system is shown in Eq. 19

$H(s)=\frac{0.00020}{0.0000010 s+1}$

The transfer function obtained is discretized by sampling interval of $10 \mathrm{sec}$ to convert it in discretetime state-space model as shown in Eqs. 20 and 21:

$x_{m}(k)=\left[\begin{array}{ccc}1 & 0 & 0 \\ 1 & 0 & 0 \\ 0 & 0 & 0\end{array}\right] x_{m}(k)+\left[\begin{array}{c}0.0200 \\ 0 \\ 0\end{array}\right] u(k)$

$y(k)=\left[\begin{array}{lll}1 & 0 & 0\end{array}\right] x_{m}(k)$
Table 2: Parameters of liquid tank for level control

\begin{tabular}{ccc}
\hline Number & Parameter & Value \\
1 & Cross sectional Area & $\mathrm{A}=100 \mathrm{~cm}^{2}$ \\
2 & Density of Water & $\mathrm{P}=1000 \mathrm{~kg} / \mathrm{m}^{3}$ \\
3 & Gravitational Acceleration & $\mathrm{g}=9.8 \mathrm{~ms}^{2}$ \\
\hline
\end{tabular}

\subsection{Optimization}

MPC optimizes the control vector $\Delta U$ by minimizing the cost function in presence ofconstraints as shown in Eq. 22 (Aslam et al., 2016b).

$J=\left(R_{S}-Y\right)^{T}\left(R_{S}-Y\right)+\Delta U^{T} \bar{R} \Delta U$

Where the first term in Eq. 22 reduces the difference between the set-point and the output, while second term gives importance to change in control signal $\Delta \mathrm{U}$ magnitude. $\overline{\mathrm{R}}$ is a diagonal matrix and $\bar{R}=r_{w} I_{N_{c} \times N_{c}} \cdot r_{w} \geq 0$ is a user defined tuning parameter for desired closed loop performance. When $r_{w}=0$ is used in cost function no importance is given to change in control signal and for large value of $r_{w}$ results in careful attention to change in control signal. The optimization of control vector is shown in Eq. 23.

$\Delta U=\left(\phi^{T} \phi+\bar{R}\right)^{-1} \phi^{T}\left(R_{S} F x\left(k_{i}\right)\right)$

where $\mathrm{F}$ and $\phi$ are the vectors shown below

$F=\left[\begin{array}{c}C A \\ C A^{2} \\ \dot{\gamma} \\ C A^{N_{p}}\end{array}\right]$ and $\phi=$
$\left[\begin{array}{cccccc}C B & 0 & 0 & 0 & \ldots & 0 \\ C A B & C B & 0 & 0 & \ldots & 0 \\ \vdots & C A B & C B & 0 & \ldots & 0 \\ C A^{N_{p}-1} B & C A^{N_{p}-2} B & C A^{N_{p}-3} B & \ldots & \ldots & C A^{N_{p}-N_{c}} B\end{array}\right]$

There are three major types of constraints two of them are applied at the control signal and change in control signal respectively while, the third one is applied at the output of the system. These constraints are of the form given below (Aslam et al., 2016c).

$\Delta U^{\min } \leq \Delta U(k) \leq \Delta U^{\max }$

$u^{\min } \leq u(k) \leq u^{\max }$

$y^{\min } \leq y(k) \leq y^{\max }$

Traditionally, the constraints are imposed on the whole control vector and all constraints are expressed in terms of vector $\Delta U$ as shown in Eq. 25 .

$$
\begin{aligned}
& {\left[\begin{array}{c}
u\left(k_{i}\right) \\
u\left(k_{i}+1\right) \\
u\left(k_{i}+2\right) \\
\vdots \\
u\left(k_{i}+N_{c}-1\right)
\end{array}\right]=\left[\begin{array}{c}
1 \\
1 \\
1 \\
\vdots \\
1
\end{array}\right] u\left(k_{i}-1\right)+} \\
& {\left[\begin{array}{ccccc}
1 & 0 & 0 & \ldots & 0 \\
1 & 1 & 0 & \ldots & 0 \\
1 & 1 & 1 & \ldots & 0 \\
\vdots & \vdots & \vdots & \ldots & 0 \\
1 & 1 & \ldots & 1 & 1
\end{array}\right]\left[\begin{array}{c}
\Delta u\left(k_{i}\right) \\
\Delta u\left(k_{i}\right)+1 \\
\Delta u\left(k_{i}\right)+2 \\
\vdots \\
\Delta u\left(k_{i}\right)+N_{c}-1
\end{array}\right]}
\end{aligned}
$$


Rearranging the above equation in a compact matrix form by relating $\mathrm{C}_{1}$ and $\mathrm{C}_{2}$ to appropriate matrices and subject to constraints for control signal as shown in Eqs. 26a and 26b.

$-\left(C_{1} u\left(k_{i}-1\right)+C_{2} \Delta U\right) \leq-U^{\min }$
$\left(C_{1} u\left(k_{i}-1\right)+C_{2} \Delta U\right) \leq U^{\max }$

where, $U^{\min }$ and $U^{\max }$ are column vectors with $\mathrm{N}_{c}$ elements of $\mathrm{u}^{\min }$ and $\mathrm{u}^{\max }$ similarly for change in control signal constraints are (Eq. 27)

$-\Delta U \leq-\Delta U^{\min }$

$\Delta U \leq \Delta U^{\max }$

Again $\Delta U^{\min }$ and $\Delta U^{\max }$ are column vectors with $\mathrm{N}_{\mathrm{c}}$ elements. Similarly, output constraints are presented in terms of $\Delta U$ (Eq. 28)

$Y^{\min } \leq F x\left(k_{i}\right)+\phi \Delta U \leq Y^{\max }$

After minimizing the cost function for optimization of control vector, $\Delta U$ is subjected to the inequality constraints by Eq. 29

$\left[\begin{array}{l}M_{1} \\ M_{2} \\ M_{3}\end{array}\right] \Delta U \leq\left[\begin{array}{l}N_{1} \\ N_{2} \\ N_{3}\end{array}\right]$

where,

$M_{1}=\left[\begin{array}{c}-C_{2} \\ C_{2}\end{array}\right] ; N_{1}=\left[\begin{array}{c}-U^{\min }+C_{1} u\left(k_{i}-1\right) \\ U^{\max }-C_{1} u\left(k_{i}-1\right)\end{array}\right] ;$

$M_{2}=\left[\begin{array}{c}-1 \\ 1\end{array}\right] ; N_{2}=\left[\begin{array}{c}-\Delta U^{\min } \\ \Delta U^{\max }\end{array}\right]$;

$M_{3}=\left[\begin{array}{l}\phi \\ \phi\end{array}\right] ; N_{3}=\left[\begin{array}{c}-Y^{\min }+F x\left(k_{i}\right) \\ Y^{\max }-F x\left(k_{i}\right)\end{array}\right]$

Transform the above equation in more compact form as shown in equation below and use it to find an optimized control without violating the constraints (Eq. 30).

$M \Delta U \leq \gamma$

Where, $\mathrm{M}$ is a vector of constraints with its rows equal to number of constraints and columns equal to dimension of $\Delta U$ (Aslam et al., 2016c).

\section{Simulations based analysis}

PLC is a special form of microprocessor- based controller as it uses programmable memory to store instructions and to implement functions such as logic, sequencing, timing, counting and arithmetic in order to control processes. PLCs have been an integral part of factory automation and industrial processes control for decades. They control a wide variety of applications from simple lightning functions to complex environmental systems and complex chemical plants. T also provides variety of analog and digital input output (I/O) interfaces, signal processing capability, data conversion and various communication protocols. The working operation and basic features of used PLC is elaborated in following section

\subsection{Basic PLC operation and specification}

The basic operation of PLC is very simple and based on the Scan Cycle loop. It consists of four steps input scan, program scan, output scan and housekeeping as shown in Fig. 4 (Netto and Bagri, 2013). The housekeeping step is executed at both start and end of operation cycle by self-checking the hardware and software to rectify or indicate any fault. In input scan PLC looks at each input card to determine if it is $\mathrm{ON}$ or $\mathrm{OFF}$ and saves this information in data table for use in next step. This makes the process faster and avoids cases where an input changes from the start to the end. In program scan PLC executes the instruction one at a time and finally in output scan after execution of program output registers are updated in this way PLC performs operation. The features of Delta DVP20EX2 module are summarized in Table 3 .

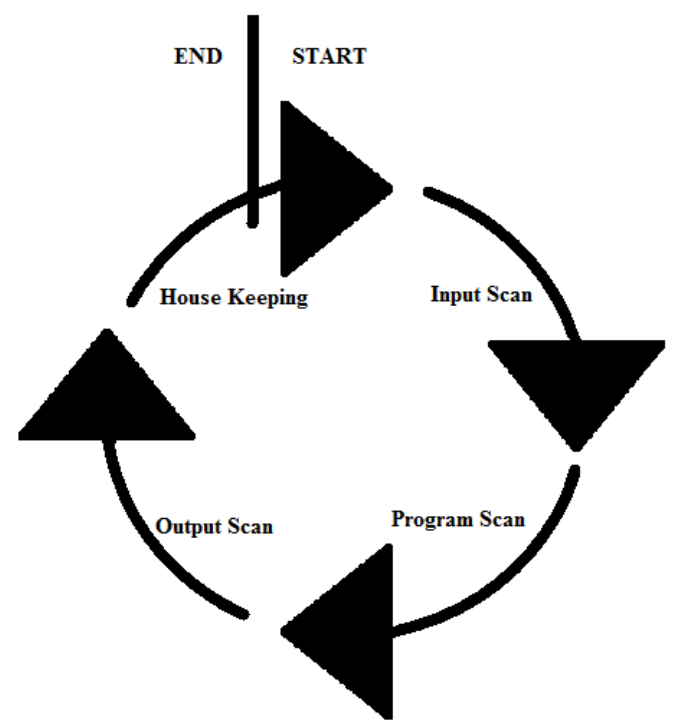

Fig. 4: PLC operational cycle

Table 3: Internal parameters of delta PLC

\begin{tabular}{ccc}
\hline Number & Specification & Quantity \\
\hline 1 & Analog Inputs & 3 \\
2 & Digital Inputs & 16 \\
3 & Data Registers & 1500 \\
4 & Internal relays & 1200 \\
5 & Counters & 200 \\
6 & Timers & 200 \\
7 & Channel Resolution & $24 \mathrm{bit}$ \\
\hline
\end{tabular}

\section{Simulation results}

Simulations of MPC for temperature and level control of water are developed by first acquiring the state-space model (SSM) of water tank then include the design parameters $\mathrm{N}_{c}, \mathrm{~N}_{\mathrm{p}}$, reference point and constraints. Finally, the cost function subject to constraints is minimized to optimize control vector which is further applied to SSM to get predicted output and this procedure continues until the desired set point is achieved. Flow chart of MPC implementation is shown in Fig. 5. 


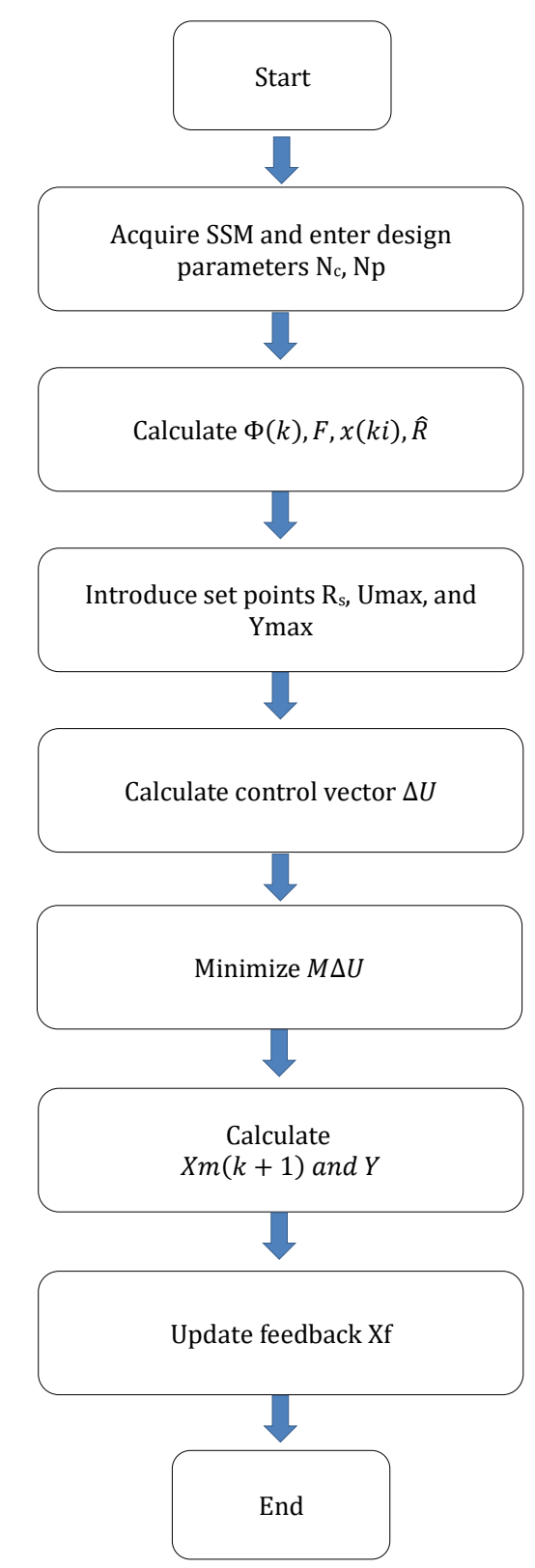

Fig. 5: Flow chart of MPC implementation

\subsection{Temperature control simulation results}

Results of temperature control of the water are presented at three set-points $50{ }^{\circ} \mathrm{C}, 60^{\circ} \mathrm{C}$ and $70{ }^{\circ} \mathrm{C}$ respectively in Fig. 6. The simulation results have shown that MPC effectively regulates the temperature at desired set-points.

\subsection{Level control simulation results}

Results of level control of the water are also presented at three set-points $250 \mathrm{ml}, 500 \mathrm{ml}$ and 1000mlin Fig. 7. The simulation results have shown that MPC effectively regulates the water level at desired set-points.

\section{Hard results}

The hardware implementation consists of Human Machine Interface (HMI) for input and Output results display, PLC for processing MPC, Water process tank as plant, sensors for feedback and a power supply. The PLC receives the actual temperature from Process tank as feedback from temperature sensor (pt100) and then PLC uses MPC algorithm to generate Pulse Width Modulation (PWM) to control the power of immersion road to regulate temperature. once the temperature meets desired value then filling of bottles start and MPC generates PWM pulses for PWM-controlled flow valve to regulate the water level and the weight cells ends the feedback to PLC to compute the required PWM to regulate desired water levels.

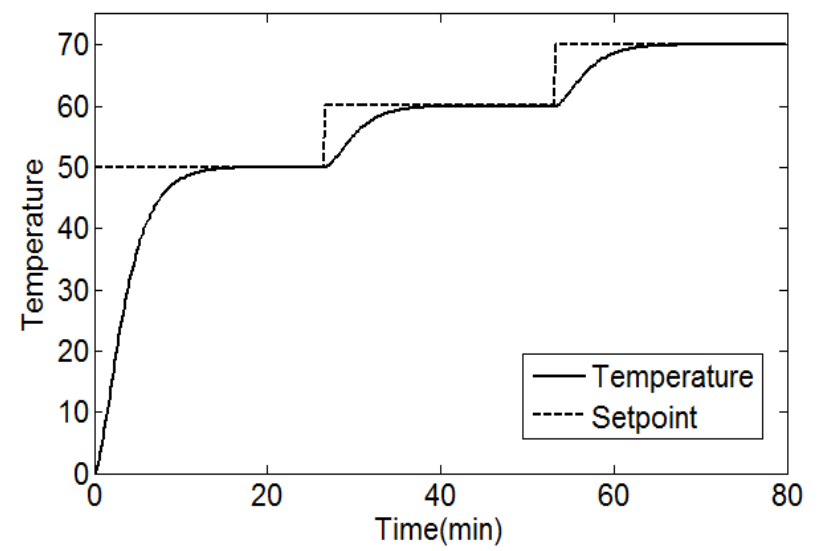

Fig. 6: Temperature control simulation

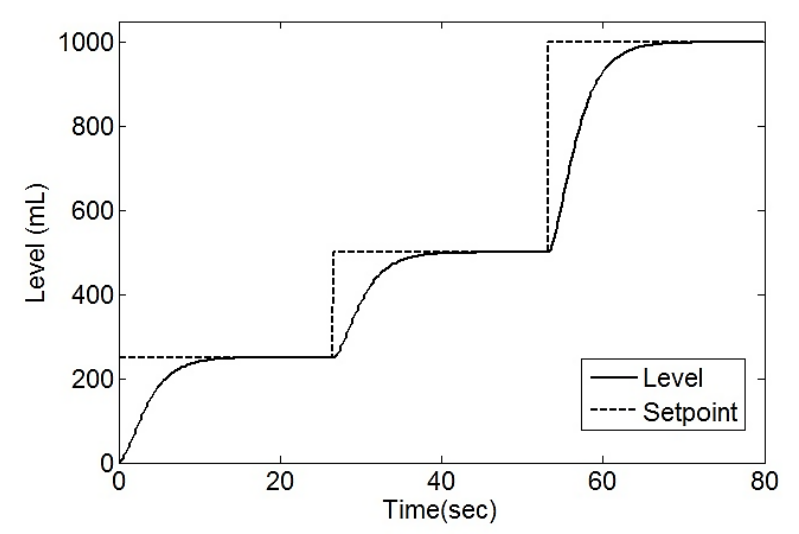

Fig. 7: Level control simulation

\subsection{Block diagram}

The Block Diagram of the industrial process control is shown in Fig. 8. It consists of an input transducer to transform the desired input reference to a form compatible with the PLC so, it can generate required PWM for PWM based control valve using MPC algorithm. Output transducers measures temperature and level and convert it into the form used by the controller and according to these feedbacks controller calculate PWM using MPC and display unit shows the result of reference value and actual values of these two parameters.

\subsubsection{Human-Machine Interface (HMI)}

Human-Machine interface (HMI) is a software application of PLC used to give commands to PLC 
and it is also used to display the output results of a system. In this industrial process automation HMI is used to give different set-points of temperature and water level and is also used to display the results of regulated variables. .

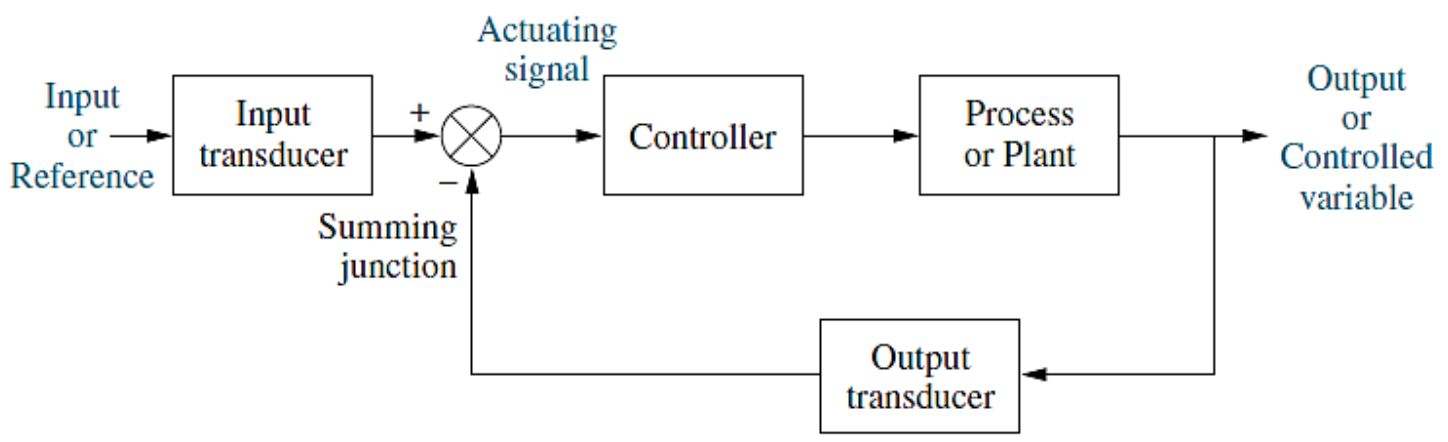

Fig. 8: Block diagram of system

\subsubsection{Programmable Logic Controller (PLC)}

DVP20EX2 is a $2^{\text {nd }}$ generation Programmable Logic Controller. There are number of programming languages of PLC. Ladder logic is used in this research work to implement MPC to regulate both temperature and level of water as an application of industrial process control

\subsubsection{Process plant}

The water tank is used in this project as a system model for regulation of temperature with heating immersion rod of 2000 Watt and filling bottles of different volumes are used for regulation of water level with PWM based flow control valve.

\subsubsection{Sensors}

Pt100 is used as an output transducer for transmitting tank's temperature back to controller. It is a Platinum resistance thermometer (PRT) that offers excellent accuracy over a wide temperature range (from -200 to $+850{ }^{\circ} \mathrm{C}$ ). Relationship between temperature and resistance is approximately linear over a small temperature range. A weight cells is a transducer which converts force into a measurable electrical output, strain gage based load cell is used in this project. Strain gage load cell have accuracy within $0.03 \%$ to $0.25 \%$ of full scale. In case of water 1 liter $=1 \mathrm{~kg}$ so, it is used to transmit the level of water in bottles to controller.

\subsection{PLC flowchart}

Real-time implementation of MPC on PLC is done by first initializing the PLC then providing temperature and level set-points from HMI. After that MPC for temperature and level control is implemented by using Ladder Logic. Finally, PWM is generated to regulate the power of both heating rod and flow control valve and indirectly control temperature and level. PLC implementation flow chart is shown in Fig. 9.

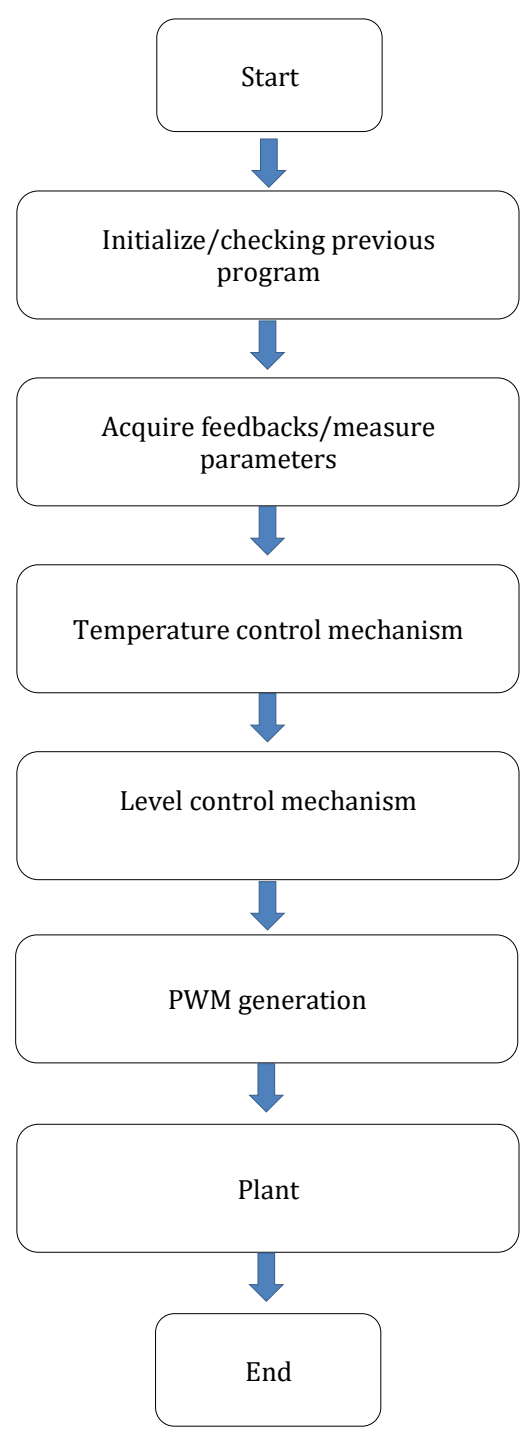

Fig. 9: Flow chart of PLC operation

\subsection{Results}

The combined hardware results for temperature and level control are presented in three cases on HMI. They are $60^{\circ} \mathrm{C}$ and $500 \mathrm{~mL}, 70^{\circ} \mathrm{C}$ and $1000 \mathrm{~mL}$ and $80^{\circ} \mathrm{C}$ and $1500 \mathrm{~mL}$. 


\subsection{1. $60^{\circ} \mathrm{C}$ and $500 \mathrm{ml}$}

MPC is implemented on PLC to initially regulate the temperature at $60^{\circ} \mathrm{Cin}$ water tank then filling of bottles start and $500 \mathrm{ml}$ bottle is filled. The results are shown in Fig. 10.

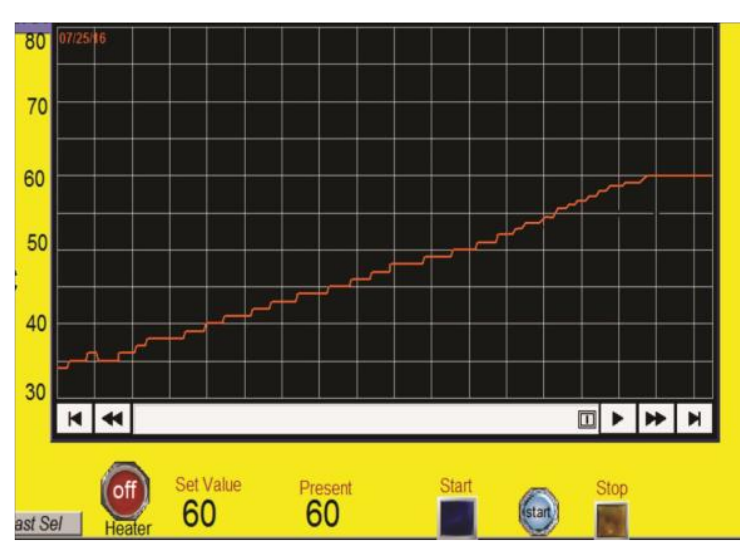

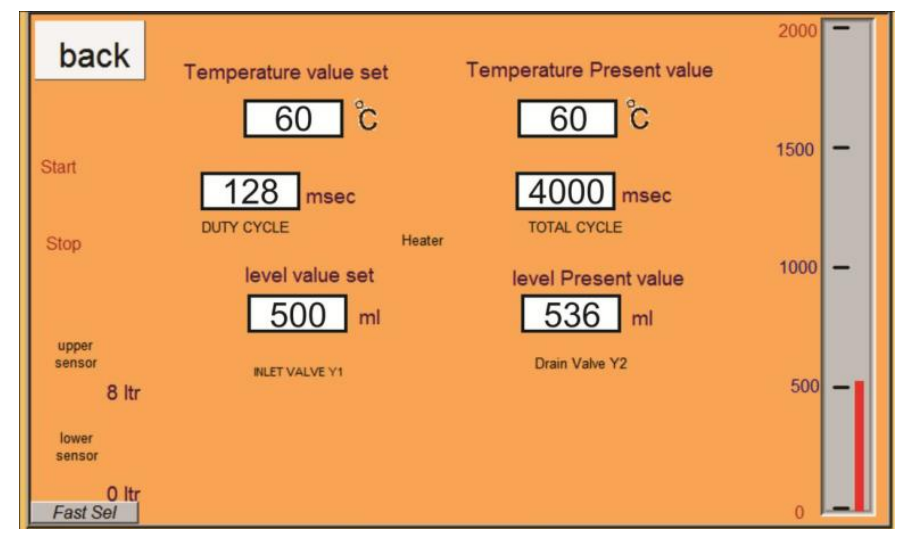

b

Fig. 10: Temperature and level regulation at $60^{\circ} \mathrm{C}$ and $500 \mathrm{~mL}$

Fig. 10a shows that MPC effectively regulates the desired temperature and Fig. 10b shows both temperature and level are regulated. Level has some steady state error and it is due to the initial empty bottle weight of 32 grams. HMI results also show the duty cycle of $128 \mathrm{msec}$ after reaching the desired temperature.

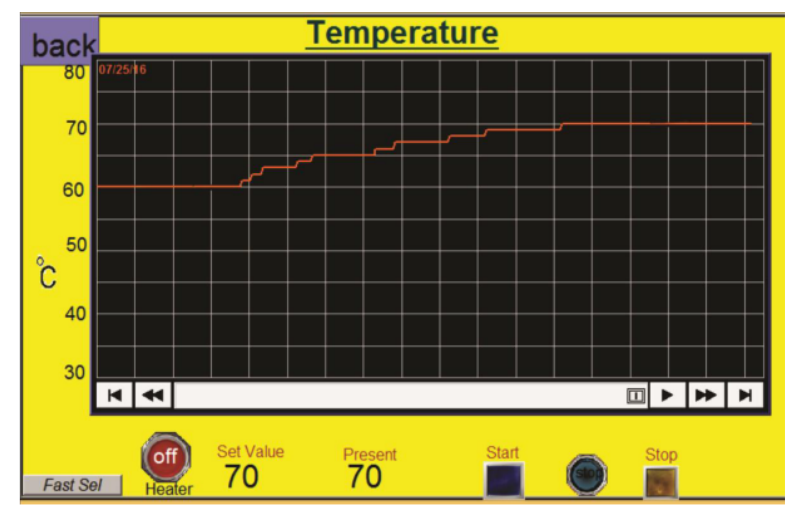

Fig. 11: Temperature and Level regulation at $70^{\circ} \mathrm{C}$ and $1000 \mathrm{ml}$

Fig. 11a shows that MPC effectively regulates the desired temperature and Fig. $11 \mathrm{~b}$ shows both temperature and level are regulated. Level has some steady state error and it is due to the initial empty bottle weight of 32 grams. HMI results also show the duty cycle of $202 \mathrm{msec}$ after reaching the desired temperature

\subsection{3. $80^{\circ}$ and $1500 \mathrm{ml}$}

MPC is implemented to regulate the temperature at $80^{\circ} \mathrm{C}$ and fill bottle at $1500 \mathrm{ml}$. The results are shown in Fig. 12. Fig. 12a shows that MPC effectively regulates the desired temperature and Fig. $12 \mathrm{~b}$ shows both temperature and level are regulated. Level has some steady state error and it is due to the initial empty bottle weight of 32 grams. HMI results also show the duty cycle of $237 \mathrm{msec}$ after reaching the desired temperature.

\subsection{2. $70^{\circ}$ and $1000 \mathrm{ml}$}

MPC is implemented to regulate the temperature at $70^{\circ} \mathrm{C}$ and fill bottle at $1000 \mathrm{ml}$. The results are shown in Fig. 11.

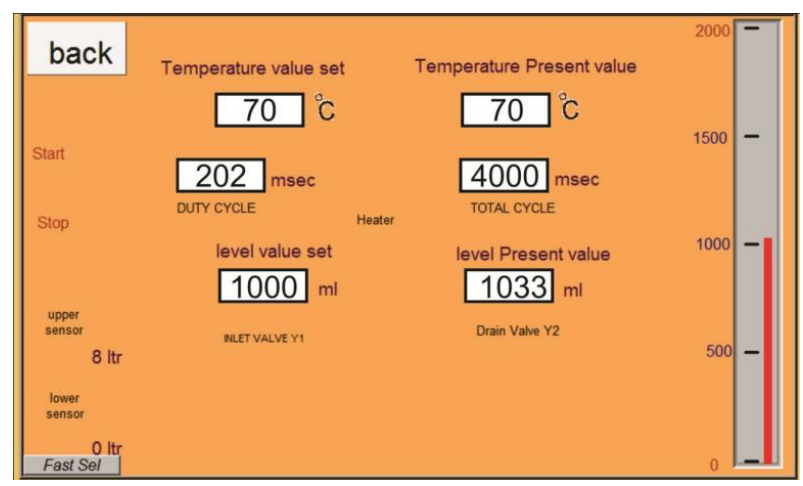

b

\section{Conclusion}

In this research work MPC is implemented on PLC for temperature and level regulation of water in tank and bottles respectively. MPC is an optimization based control technique and provides effective controlling in comparison to other control techniques but it has high computational burden so PLC is best solution for MPC in industrial process control. First simulations of MPC are presented in MATLAB for both temperature and level control at different set-points and MPC convincingly regulate both variables. Finally, MPC is real-time implemented on PLC to regulate both temperature and level and results are displayed on HMI. The results have shown that MPC effectively regulates temperature of water in tank and level in bottles at different set-points. 


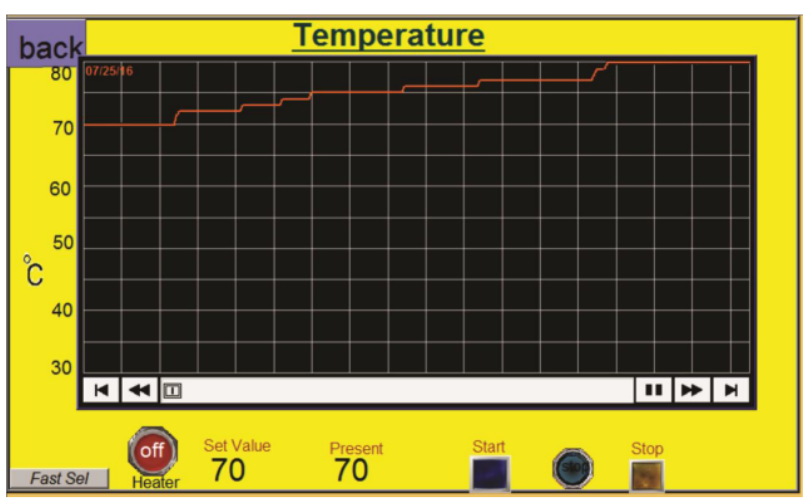

a

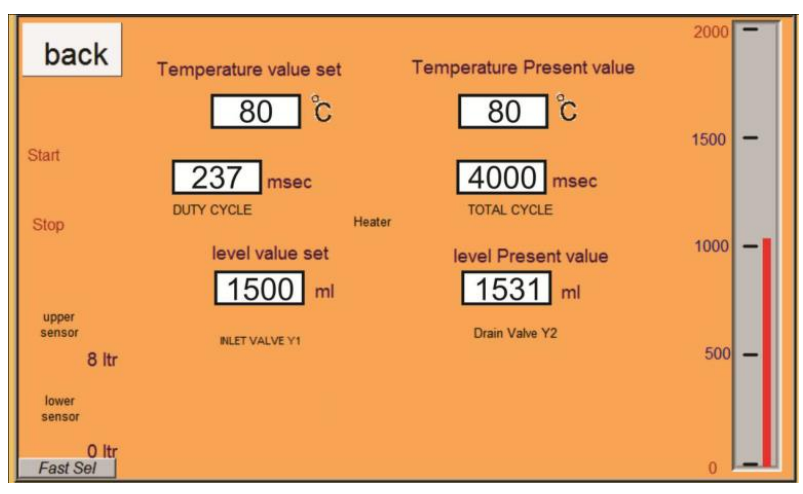

b

Fig. 12: Temperature and Level regulation at $80^{\circ} \mathrm{C}$ and $1500 \mathrm{ml}$

\section{References}

Aslam S, Hannan S, and Haider A (2016b). Effect of laguerre function parameters on MPC performance for speed control of a DC motor. Journal of Control Engineering and Technology, 6(1): 1-13.

Aslam S, Hannan USS, and Zafarm W (2016a). Implementation of PID on PIC24F series microcontroller for speed control of a DC motor using MPLAB and Proteus. Advances in Science and Technology Research Journal, 10(31): 40-50.

Aslam S, Hannan USS, and Zafarm W (2016c). Temperature control of water-bath system in presence of constraints by using MPC. International Journal of Advanced and Applied Sciences, 3(12): 62-68.

Colla M, Leidi T, and Semo M (2009). Design and implementation of industrial automation control systems: A survey. In the IEEE 7th International Conference on Industrial Informatics. IEEE, Wales, UK: 570-575. https://doi.org/10.1109/INDIN. 2009.5195866

Huyck B, Ferreau HJ, Diehl M, De Brabanter J, Van Impe JF, De Moor B, and Logist F (2012). Towards online model predictive control on a programmable logic controller: Practical considerations. Mathematical Problems in Engineering, 2012: Article ID 912603, 20 pages. http://dx.doi.org/10.1155/ 2012/912603
Lashin MM (2014). Different applications of programmable logic controller (PLC). International Journal of Computer Science, Engineering and Information Technology, 4(1): 27-32.

Li SE, Jia Z, Li K, and Cheng B (2013). Scale reduction based efficient model predictive control and its application in vehicle following control. In the IEEE 16th International Conference on Intelligent Transportation Systems, IEEE, The Hague, Netherlands: 1266-1271. https://doi.org/10.1109/ITSC.2013. 6728405

Liuping (2009). Model predictive control system design and implementation using MATLAB. Springer Science \& Business Media, London, UK.

Maciejowski J (2002). Predictive control: With constraints, Prentice Hall, New Jersey, USA. .

Netto R and Bagri A (2013). Programmable logic controllers. International Journal of Computer Applications, 77(11): 27 31.

Qin SJ and Badgwell TA (2003). A survey of industrial model predictive control technology. Control Engineering Practice, 11(7): 733-764.

Rathore RS, Sharma AK, and Dubey HK (2015). PLC based PID implementation in process control of temperature flow and level. International Journal of Advanced Research in Engineering and Technology, 6(1): 9-26. 